\title{
Near-rings in which each element is a power of itself
}

\author{
Howard E. Bell
}

Let $R$ denote a near-ring such that for each $x \in R$, there exists an integer $n(x)>1$ for which $x^{n(x)}=x$. We show that the aditive group of $R$ is commutative if $0 . x=0$ for all $x \in R$ and every non-trivial homomorphic image $\vec{R}$ of $R$ contains a non-zero idempotent $e$ commuting multiplicatively with all elements of $\bar{R}$. As the major consequence, we obtain the result that if $R$ is distributively-generated, then $R$ is a ring - a generalization of a recent theorem of Ligh on boolean near-rings.

\section{Introduction}

In [6], Ligh proved that a distributively-generated boolean near-ring is a ring and asked whether the same can be said of distributivelygenerated near-rings satisfying the identities $x^{p}=x$ and $p x=0$, where $p$ is a prime. We give here an affirmative answer to this question, and we obtain some more general results on additive commutativity in near-rings in which $x^{n(x)}=x$. The major theorems are

THEOREM 1. Let $R$ be a non-trivial near-ring satisfying the following properties:

(i) $0 . x=0$ for all $x \in R$;

(ii) for each $x \in R$, there exists an integer $n(x)>1$ such that $x^{n(x)}=x$;

Received 21 February 1970. 
(iii) every non-trivial homomorphic image of $R$ contains a non-zero central idempotent.

Then the additive group of $R$ is commutative.

THEOREM 2. Let $R$ be a distributively-generated neor-ring such that for each $x \in R$ there is an integer $n(x)>1$ for which $x^{n(x)}=x$. Then $R$ is a commutative ring.

\section{Definitions and preliminary results}

Our definitions of near-ring, distributive element, distributivelygenerated near-ring, and ideal are as in [6]. A near-ring ideal $P$ will be called completely prime if $a b \in P$ implies $a \in P$ or $b \in P$. An element $a$ of the near-ring $R$ will be called central if $x a=a x$ for all $x \in R$.

The left distributive law implies

$$
x .0=0 \text { for all } x \in R
$$

and

$$
x(-y)=-x y \text { for all } x, y \in R \text {; }
$$

moreover, if $d$ is a distributive element of $R$, we have

$$
(-x) d=-x d \text { for all } x \in R \text {. }
$$

Property (2) permits left cancellation of elements which are not zero-divisors; and from ( 1 ) it follows that in near-rings satisfying (i), the notion of nilpotent element may be borrowed from ring theory, with nilpotent elements behaving as we would expect. In particular, we have the readily-proved

LEMMA 1. If $R$ is a necr-ring satisfying (i) and having no non-zero nilpotent elements, then $a b=0$ implies that $b a=0$ and that $a r b=0$ for all $r \in R$.

We shall refer to the second conclusion of this lemma as IFP (insertion-of-factors property).

The elementary proofs of the " $x^{n}=x$ theorem" for rings use the fact that in rings with no non-zero nilpotent elements, idempotents are 
central. This result does not extend to near-rings satisfying (i) (note counterexamples in [2]); however, we obtain a partial generalization as follows :

LEMMA 2. Let $R$ be a near-ring satisfying (i) and having no non-zero nilpotent elements. Then we have

(A) every distributive idempotent is central;

(B) for every idempotent $e$ and every element $x \in R$, $e x^{2}=(e x)^{2}$;

(c) if $R$ has a multiplicative identity element, then all idempotents are central.

Proof. We first show that for each $x \in R$ and idempotent $e$, $x e=e x e$. Since $e(x e-e x e)=0$, Lemma 1 guarantees that $(x e-e x e) e=0=(x e-e x e) e(-x e) ;$ hence, we have $(x e-e x e)^{2}=(x e-e x e) x e+(x e-e x e)(-e x e)=0$, so that $x e-e x e=0$.

If $e$ is a distributive idempotent, we also have $(e x-e x e) e=e x e+(-e x e) e ;$ hence by (3) (ex-exe)e $=0$. It follows that $e(e x-e x e)=e x-e x e=0$; and the proof of $(A)$ is complete.

To establish (B), note that for any idempotent $e, x e(x-e x)=0$, so that by IFP we get $e x(x-e x)$ nilpotent and hence zero.

To establish (C), we need only show that if $R$ has 1 , then $e x=e x e$ for all $x \in R$ and arbitrary idempotents $e$. Now $e(1-e)=0$, so $(1-e) e=0$ as well; moreover, $e(e x-e x e)=e x-e x e$ and $e x(1-e)=e x-e x e$. Therefore, $(e x-e x e)^{2}=e x(1-e) e(e x-e x e)=0=e x-e x e$.

The standard proofs of the " $x^{n}=x$ theorem" for rings involve ideals which are not easily shown to be normal subgroups of $R^{+}$; we overcome this obstacle by use of a kind of annihilator ideal introduced in [1].

LEMMA 3. Let $R$ be a non-trivial near-ring satisfying (i) and having no non-zero nilpotent elements. Then $R$ contains a family of completely prime ideals with trivial intersection.

Proof. Since $R$ has no non-zero nilpotent elements, there must 
exist multiplicative subsemigroups which do not contain zero, and an application of Zorn's Lemma shows that any such subsemigroup is contained in a subsemigroup maximal with respect to excluding zero. Let $M$ be any such maximal subsemigroup, and define

$$
A(M)=\{x \in R \mid a x=0 \text { for at least one } a \in M\} .
$$

If $u, v \in A(M)$, there exist $a, b \in M$ such that $a u=b v=0$. By IFP, we then have $a b u=0$, and thus $a b(u-v)=0$; moreover, for arbitrary $x \in R, a(x+u-x)=0$, so $A(M)$ is a normal subgroup of $R^{+}$. Also, if $x, y \in R$, we have $a x u=0$ and $a[(x+u) y-x y]=a(x+u) y-a x y=(a x+a u) y-a x y=(a x+0) y-a x y=0$; hence $A(M)$ is an ideal.

Now if $x \notin M$, the multiplicative subsemigroup generated by $M$ and $x$ must contain 0 ; and since $R$ has no non-zero nilpotent elements, some finite product containing $x$ as at least one factor and having at least one factor from $M$ must be zero. Repeated application of IFP establishes the existence of an $m \in M$ such that $m x$ is nilpotent and hence 0 . Therefore the set-theoretic complement of $A(M)$ is $M$, and $A(M)$ is a completely prime ideal. Clearly every non-zero element of $R$ is excluded from at least one of the ideals $A(M)$.

\section{Proofs of Theorems 1 and 2 and some corollaries}

Proof of Theorem 1. A near-ring satisfying (i) and (ii) obviously has no non-zero nilpotent elements, hence Lemma 3 applies. For each $P=A(M)$, the near-ring $\bar{R}=\frac{R}{P}$ satisfies $(i)$ and $(i i)$, has no zero-divisors, and contains a non-trivial central idempotent $e_{0}$. From part (B) of Lema 2, we see that every idempotent of $\bar{R}$ is a left identity element, hence $e_{0}$ is the only non-zero idempotent and is an identity element. Now $a^{n}=a$ implies $a^{n-1}$ is idempotent, hence non-zero elements in $\bar{R}$ have inverses and $\bar{R}$ is then a near-field. Thus $\bar{R}$ has commutative addition $[5,7]$; and additive commutators in $R$ lie in each of the completely prime ideals $A(M)$, hence are zero.

Proof of Theorem 2. All distributively-generated near-rings satisfy 
(i). Moreover, if $a$ is a distributive element and $a^{n}=a$, then $a^{n-1}$ is a distributive idempotent, which is central by part (A) of Lemma 2. Thus, by Theorem 1, $R^{+}$is commutative. But by a theorem of Fröhlich [3, p. 93], additive commutativity in a distributively-generated near-ring $R$ implies that $R$ is a ring. That $R$ is also a commutative ring is the well-known $" x^{n}=x$ theorem" of Jacobson [4].

Two corollaries of Theorem 1 are

THEOREM 3. Let $R$ be a near-ring with identity satisfying (i) and (ii). Then $R^{+}$is corrmutative.

THEOREM 4. Let $R$ be a finite near-ring; and suppose $R$ is embeddable in a near-ring with identity which satisfies (i) and has no non-zero nilpotent elements. Then $R^{+}$is commutative.

Theorem 3 is obvious; Theorem 4 follows from Theorem 1 and part (c) of Lemma 2 once we note that a finite near-ring with (i) and without nilpotent elements satisfies (ii).

\section{Remarks}

In the class of near-rings satisfying ( $i$ ) and ( $i i)$, condition ( $i i i)$ is sufficient for additive commativity; but it is not necessary, as we see by considering [2], example 53 with additive group $z_{6}$. Lemma 2 and Theorems 3 and 4 point out an apparent difference in behaviour depending on whether $R$ does or does not have an identity element. This difference is real, as is 'shown by [2], example 34 with additive group $S_{3}$.

\section{References}

[1] Howard E. BelI, "Duo rings: some applications to commutativity theorems", Canad. Math. BuzZ. 11 (1968), 375-380.

[2] James R. Clay, "The near-rings on groups of low order", Math. 2. 104 (1968), 364-371.

[3] A. Fröhlich, "Distributively generated near-rings, (I. Ideal Theory)", 
Proc. London Math. Soc. (3), 8 (1958), 76-94.

[4] I.N. Herstein, "An elementary proof of a theorem of Jacobson", Duke Math. J. 21 (1954), 45-48.

[5] Steve Ligh, "On distributively generated near-rings", Proc. Edinburgh Math. Soc. 16 (1969), 239-243.

[6] Steve Ligh, "On boolean near-rings", Bul2. Austral. Math. Soc. 1 (1969), 375-379.

[7] J.L. Zemmer, "The additive group of an infinite near-field is abelian", J. London Math. Soc. 44 (1969), 65-67.

Brock University,

St Catharines,

Ontario, Canada. 